International Journal of

BioScience and Applications

\title{
A narrative review and case report of the use of pulsed radiofrequency in pain management: Intra- articular application
}

\author{
Ju-Hwan Lee', Dong-Ji Ahn ${ }^{1}$, and Yeon-Dong Kim ${ }^{1,2}$ \\ ${ }^{1}$ Department of Anesthesiology and Pain Medicine, Wonkwang University School of Medicine, Wonkwang \\ University Hospital, Iksan, Korea \\ ${ }^{2}$ Jesaeng-Euise Clinical Anatomy Center, Wonkwang University School of Medicine, Iksan, Korea
}

\begin{abstract}
Background/Objectives: Pulsed radiofrequency(RF) method has been introduced as a way to provide longer-term pain relief in peripheral nerve areas especially in the management of chronic pain. It is a new treatment method that complements the problems of conventional RF treatment. Methods/Statistical analysis: We describe the clinical use of pulsed RF in a patient with degenerative osteoarthritis of the knee and review of the literature related to the use of clinical practice. Findings: It showed updated information regarding the science of pulsed RF by highlighting basic science, animal study, and clinical data and to explore further the validity of the concept behind the application. Improvements/Applications: Pulsed RF may promise as an analgesic modality to treat a variety of conditions.
\end{abstract}

\section{Index Terms}

chronic pain, degeneration, nerve block, osteoarthritis, pulsed radiofrequency, review

Corresponding author : Yeon-Dong Kim

kydpain@hanmail.net

- Manuscript received July 1, 2020.

- Revised August 10, 2020 ; Accepted September 2, 2020.

- Date of publication September 30, 2020.

(c) The Academic Society of Convergence Science Inc.

2619-8363 $\odot 2020$ IJBSA. Personal use is permitted, but republication/redistribution requires IJBSA permission. 


\section{INTRODUCTION}

Degenerative osteoarthritis (OA) is a disease where the joint cartilage wears out accompanied by hardening of the inferior cartilage and the bone around the joint. It causes joint deformations, and it is most commonly seen in older people. It is a common cause of pain in the knee. As the elderly population increases, the number of patients with degenerative $\mathrm{OA}$ is also increasing proportionally, increasing the social interest due to medical expenses, and also leading to important diseases being considered for treatment, which is dominated by elderly patients. Conservative treatments such as drug therapy and physical therapy are performed for patients with mild to moderate pain, and surgical treatments such as intra-articular injection or nerve block are performed for patients not responding to treatment. In severe cases, osteotomy or total knee joint replacement is performed [1].

Pain secondary to degenerative OA could be controlled by epidural blocks, trigger point injection and local anesthetic blocks in the peripheral nerves of the knee joint depending on the area of pain. Recently, the pulsed radiofrequency (pulsed RF) lesioning method has been introduced as a way to provide long-term pain relief in the peripheral nerve areas $[2,3]$. Pulsed RF is a new treatment method that complements the problems of conventional RF treatment caused by existing heat, and there are no side effects such as nerve injury or neuritis [4]. The intra-articular (IA) application of pulsed RF for pain in small and large joints is a recent development that is proven effective in many cases. Given its current uses, a review of all available pulsed RF literature is needed.

The purpose of this study is to provide updated information regarding the science of pulsed RF by highlighting basic science, discussing results of animal study and clinical data. This study also aimed to explore further the validity of the concept behind the application of pulsed RF focused on the joint pain through a case report.

\section{CASE}

A 64-year-old female visited the department of pain medicine with a chief complaint of left knee pain. She did not have any significant medical history. Knee pain gradually worsened starting 3 years ago which was not relieved by conservative treatment such as medication, physical therapy, intraarticular injection and other peripheral nerve block. $\mathrm{X}$-ray showed severe degenerative OA of the left knee with Kellgren Lawrence grade II (Fig 1). On physical examination, there was tenderness and severe pain in the medial aspect of the knee with pain score of 8 out of 10 in visual analogue scale (VAS).

Intra-articular injection of hyaluronic acid or local anesthetics showed some pain relief but it did not last long. After explanation to the patient, intra-articular pulsed RF was scheduled. The patient was prepared in the supine position. After aseptic dressing, a 22gauge, $54 \mathrm{~mm}$ RF needle with a $5 \mathrm{~mm}$ active tip was inserted into the knee joint. Fluoroscopy confirmed the appropriate position of the RF needle tip in the knee joint on the anterior and lateral image (Fig 2). Pulsed RF was applied with a pulse width of $20 \mathrm{~ms}$, at $2 \mathrm{~Hz}$ for 15 minutes at a temperature of $42^{\circ} \mathrm{C}$ using an RF generator (NeuroTherm, RDG Medical, UK). No significant event was observed during the procedure. For six months after the procedure, the patient followed up, satisfied with the pain relief and had 3 out of 10 pain score in the VAS. This showed much better pain relief than other previous treatments.

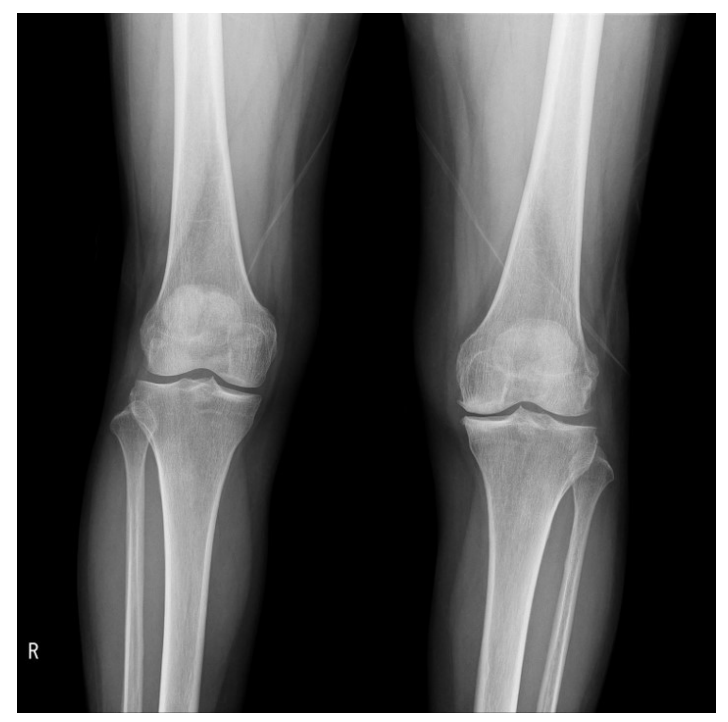

Fig. 1. Knee X-ray of the patient. It shows a degenerative osteoarthritis.

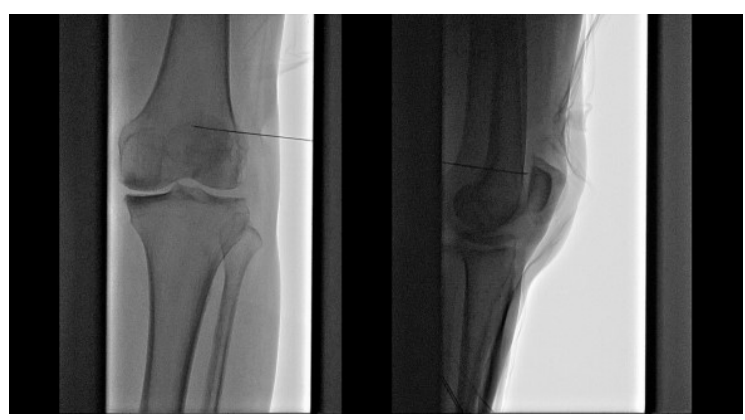


Fig. 2. Fluoroscopic confirmation of the radiofrequency needle. Intra-articular position in the knee joint on the anterior-posterior and lateral image.

\section{DisCuSSION}

The use of electric current for pain management has a long history. However, its popularity waxed and waned over time due to concerns about safety and technical improvements. Early in the 19th century, brain lesions in animals were created by direct current application, and empirical rules for quantifying lesion size based on current and time were developed.

Over the years, the concept of the clinical effect of RF by the formation of heat had not been challenged. Thermo-coagulation of nerve fibers would interfere with the conduction of nociceptive stimuli, and the pain is relieved when the nociceptive stimuli stopped reaching the spinal cord. However, there were several reasons why the role of heat was questioned. First, the RF lesioning adjacent to the dorsal root ganglion (DRG) induces only transient sensory loss in the relevant dermatome. This can be considered heat related, while the pain relief may be of much longer duration. Second, the classical concept assumes a strict configuration; the RF lesioning must be made in between the nociceptive focus and the central nervous system. Third, the role of heat was also questioned by a publication that showed no difference in the outcome when two different tip temperatures $\left(40^{\circ} \mathrm{C}\right.$ and $\left.67^{\circ} \mathrm{C}\right)$ were applied to the cervical DRG in chronic cervical radicular pain.

It is against this background that pulsed RF was developed. Pulsed RF sends pulses of electrical currents from an electrode tip to targeted nerve tissue to be applied to the area of joint pain. The pulsatile nature of the stimulation allows the tip to cool between pulses, keeping the electrode below $42^{\circ} \mathrm{C}$; the temperature level which causes damage to the surrounding tissue. The targeted nociceptive tissue is thought to sustain damage from high-density electrical fields generated during the pulsed RF, although the exact mechanism of destruction is not completely understood. Pulsed RF aims to deliver strong electric fields, while the temperature effects are kept to a minimum.

In case reports by Sluijter et al, the use of pulsed $\mathrm{RF}$ in the treatment of chronic joint pain was evaluated. Pulsed RF was used in patients in whom both conservative and other invasive treatment failed. Pain relief was achieved in each case, with a duration of effect lasting for 8-12 months [5]. More data for the use pulsed RF on other joints are as follows:

\section{Shoulder Pain}

In 2002, pulsed RF of the suprascapular nerve was first described. Rohof evaluated the effect of pulsed RF on the suprascapular nerve by performing a retrospective analysis of 37 cases with chronic shoulder pain [6]. After treatment, there was an average reduction in pain score of 4 in VAS. Kane et al. evaluated the effects of pulsed RF [7]. In 12 patients with painful arthropathy of the rotator cuff, it was reported that patients' scores on the VAS, Oxford Shoulder Score, and Constant Shoulder score at both 3 and 6 months improved compared to baseline.

\section{Hip Pain}

In 14 cases with hip pain, Kawaguchi et al found that twelve cases had $>50 \%$ pain reduction for 1 to 11 months [8]. Similar results were noted in an additional RF case report. Common diagnostic uses reported in the literature are OA, avascular necrosis of the hip, bone malignancy, fractures, and persistent post arthroplasty pain. Outcomes in several prospective studies have shown improvement of pain up to 11 months, decreased consumption of pain medication, and improved patient function. However, most published studies are case reports and retrospective studies with only 2 prospective studies [9.10].

\section{Knee}

Six publication reports showed that conventional or cooled ablative RF treatments target periarticular nerves to relieve chronic pain in the knee joint secondary to $\mathrm{OA}$ or to relieve persistent pain following surgery $[11,12,13,14]$. The main targeted anatomical structure reported in the articles were as follows:

- Sensory nerves innervating the anteromedial aspect of the knee joint capsule including the infrapatellar branch of the saphenous nerve and the terminal branch of the nerve to the vastus medialis (medial retinacular nerve)

- Periosteum of the shaft of the femur and the tibia to target the nerves innervating the knee.

A case report and four case series reported focused intra-articular pulsed RF treatments for chronic pain in the knee joint secondary to OA $[5,15,16,17]$. It showed significant pain reduction during the followup period from 1 to 12 months.

\section{Foot and Ankle, Elbow}

The approach to peripheral pain including epicondylitis, is still challenging in clinical practice. In 2002, it was suggested that pulsed RF may provide an effective treatment for less severe pain arising from lateral epicondylitis [3]. However, no 
published research defined the exact target of pulsed RF treatment in cases of epicondylitis. Pelloso et al reported a case of chronic ankle pain, wherein the patient was submitted to two sessions. They were able to achieve improvement of approximately $80 \%$ of pain, allowing improvement in daily activities [18].

\section{CONCLUSION}

The understanding of pulsed RF evolved in the past few years, despite lack of understanding on its mechanism. The focus of the investigation on the mechanisms of action of pulsed RF is moving from cellular structure to the expression of the genetic level related to pain perception. As a result, long term pain relief mechanisms are concluded to be due to the reduction of nociception signal. Further study with the animal behavior also support the evidence of the Pulsed RF in pain management. Enhancement of descending noradrenergic and serotonergic inhibitory pathways has been postulated as the main mechanism up to now.

Pulsed RF may be a promising analgesic modality to treat various pain conditions.

\section{REFERENCES}

[1] Cimmino, M. A., Parodi, M. (2005). Risk factors for osteoarthritis. Seminars in arthritis and rheumatism, 34(6 Suppl 2), 29-34.

[2] [2] Kawaguchi, M., Hashizume, K., Iwata, T., Furuya, H (2001). Percutaneous radiofrequency lesioning of sensory branches of the obturator and femoral nerves for the treatment of hip joint pain. Regional anesthesia and pain medicine, 26(6), 576-581.

[3] [3] Rohof O. J. (2002). Radiofrequency treatment of peripheral nerves. Pain practice, 2(3), 257-260.

[4] [4] Sluijter, M. E., van Kleef, M. (1998). Characteristics and mode of action of radiofrequency lesions. Current Review of pain, 2(3), 143-150.

[5] [5] Sluijter, M. E., Teixeira, A., Serra, V., Balogh, S., Schianchi, P. (2008). Intra-articular application of pulsed radiofrequency for arthrogenic pain-report of six cases. Pain practice, 8(1), 57-61.

[6] [6] Rohof, O. J. J. M. (2002). Radiofrequency treatment of peripheral nerves. Pain Practice, 2(3), 257-260.

[7] [7] Kane, T. P., Rogers, P., Hazelgrove, J., Wimsey, S., Harper, G. D. (2008). Pulsed radiofrequency applied to the suprascapular nerve in painful cuff tear arthropathy. Journal of shoulder and elbow surgery, 17(3), 436-440.

[8] [8] Kawaguchi, M., Hashizume, K., Iwata, T., Furuya, H. (2001). Percutaneous radiofrequency lesioning of sensory branches of the obturator and femoral nerves for the treatment of hip joint pain. Regional anesthesia and pain medicine, 26(6), 576-581.

[9] [9] Choi, W. J., Hwang, S. J., Song, J. G., Leem, J. G., Kang, Y. U., Park, P. H., Shin, J. W. (2011). Radiofrequency treatment relieves chronic knee osteoarthritis pain: a doubleblind randomized controlled trial. PAIN, 152(3), 481-487.

[10] Ikeuchi, M., Ushida, T., Izumi, M., Tani, T. (2011) Percutaneous radiofrequency treatment for refractory anteromedial pain of osteoarthritic knees. Pain Medicine, 12(4), 546-551.

[11] Bellini, M., Barbieri, M. (2015). Cooled radiofrequency system relieves chronic knee osteoarthritis pain: the first case-series. Anaesthesiology intensive therapy, 47(1), 30-33.

[12] Clendenen, S., Greengrass, R., Whalen, J., \& O'Connor, M. I. (2015). Infrapatellar saphenous neuralgia after TKA can be improved with ultrasound-guided local treatments. Clinical Orthopaedics and Related Research, 473(1), 119125.

[13] Menzies, R. D., Hawkins, J. K. (2015). Analgesia and improved performance in a patient treated by cooled radiofrequency for pain and dysfunction postbilateral total knee replacement. Pain Practice, 15(6), E54-E58.

[14] Kvarstein, G. (2011). A new target for radiofrequency neurotomy?. Pain, 152(3), 465-466.

[15] Karaman, H., Tüfek, A., Kavak, G. Ö., Yildirim, Z. B., Uysal, E., Celik, F., Kaya, S. (2011). Intra-articularly applied pulsed radiofrequency can reduce chronic knee pain in patients with osteoarthritis. Journal of the Chinese Medical Association, 74(8), 336-340.

[16] Masala, S., Fiori, R., Raguso, M., Morini, M., Calabria, E., Simonetti, G. (2014). Pulse-dose radiofrequency for knee osteoartrithis. Cardiovascular and interventional radiology, 37(2), 482-487.

[17] Eyigor, C., Eyigor, S., Akdeniz, S., Uyar, M. (2015). Effects of intra-articular application of pulsed radiofrequency on pain, functioning and quality of life in patients with advanced knee osteoarthritis. Journal of back and musculoskeletal rehabilitation, 28(1), 129-134.

[18] Pelloso, L. R. C. D. A., Freire, G. M. G., Ashmawi, H. A. (2012). Refractory chronic ankle pain controlled with pulsed radiofrequency: case report. Revista Dor, 13(4), 389-391. 